\title{
Failure Pressures and Drag Reduction Benefits of Superhydrophobic Wire Screens
}

\author{
D.G. Venkateshan, M.M Amrei, A.A. Hemeda, Z. Cullingsworth, J. Corbett, and H. Vahedi Tafreshi* \\ Department of Mechanical and Nuclear Engineering, \\ Virginia Commonwealth University, Richmond, Virginia 23284-3015
}

\begin{abstract}
This work presents a detailed study on the failure pressure of spray-coated superhydrophobic wire screens in terms of their geometric and wetting properties. Such information is needed in designing fluid-fluid or fluid-air separation/barrier media as well as drag reducing and self-cleaning surfaces, amongst many others. Good agreement has been observed between the results of our numerical simulations and the experimental data for failure pressure. In addition, the wetted area of the screens was calculated and used to predict their drag reduction benefits when used in a Couette flow configuration under different operating pressures. Interestingly, it was found that operating pressure in the Couette configuration does not significantly affect the drag reducing effects of the screens.
\end{abstract}

Keywords: Superhydrophobic wire screens; breakthrough pressure; wetting; air-water interface; slip length

\footnotetext{
*Address correspondence to Hooman V. Tafreshi, email: htafreshi@ vcu.edu; tel.: 804-828-9936; fax: 804-827-7030; URL: http://www.people.vcu.edu/ htafreshi/
} 


\section{INTRODUCTION}

Superhydrophobic (SHP) surfaces, often produced by incorporating single or multiscale roughness into a hydrophobic material, are known for their ability to reduce the area of contact between water and the solid surface (referred to here as the wetted area). The reduced wetted area, in turn, brings about peculiar attributes that are essential for applications such as self-cleaning [1-3] and drag-reduction [3-7] to name a few. Given the prohibitive cost associated with large-scale production of microfabricated SHP surfaces, woven screens enhanced with functional surface treatments/coatings have recently been considered as a cost-effective alternative in many applications requiring a SHP surface. SHP woven screens can be used for drag reduction [8-11], oil-water separation [12-16], self-cleaning and anti-icing [17-19], underwater protection of electronic devices [20-21], water harvesting [22], and heat transfer [23] among many other applications. There have also been interesting studies on the load-carrying properties of SHP wire screens for device manufacturing [24-28].

Performance of a SHP surface depends on the mechanical stability of the air-water interface (AWI) that forms over the non-wetting pores of the surface upon contact with water. Depending on surface geometry and hydrostatic/hydrodynamic pressure, the AWI can ingress into the space between the wires to allow the Wenzel state (fully wetted), the Cassie state (fully dry), or a series of transition states in between the two extreme states to prevail over the surface. The main forces acting on an AWI are the hydrostatic/hydrodynamic pressure and the capillary pressure (see [29-30] for more detailed information). The hydrostatic pressure at which a SHP surface starts departing from the Cassie state is referred to as the critical hydrostatic pressure (CHP) [29-30]. This definition is often used in the context of pores with sharp-edged entrance where the AWI can anchor (pin) itself to the edges of the pore. The definition is less clear when the pore entrance is round (the case with wire screens). This is because in this case, the AWI cannot anchor itself to any sharp corner, and has to conform to a shape that maintains the Young-Laplace contact angle (YLCA) at any point along the curved walls of the pore. Therefore, even at a zero hydrostatic pressure, it is hard to define a fully dry (Cassie) state. Obviously, the AWI moves 
further down into the pore in response to any increase in the hydrostatic pressure [17, 31-34]. For the lack of a better alternative, we define CHP for a pore with round entrance, to be the hydrostatic pressure at which the AWI moves down into the pore such that the pore's capillary pressure reaches its maximum value (referred to here as the breakthrough pressure) denoted with $P_{b r k}$ (see [17, 31-34] for more detailed information). Transition to the Wenzel state can also occur if the AWI touches the bottom of the pore under pressure. This has been identified in the literature as failure due to AWI sagging or the lack of "robustness height" $[31,35]$. For applications in which a wire screen is used as a coating placed on a surface (e.g., [9-12, and 18-19]), we consider sagging to be the failure mechanism and the pressure associated with this pressure is shown with $P_{s a g}$. On the other hand, for applications in which a wire screen operates as a barrier between two different fluids or two different compartments (e.g., [13-17, 20-22, 24-28]), we consider failure to be the condition where the pressure over the screen exceeds the breakthrough pressure.

In the remainder of this paper, we first present our numerical approach for predicting the wetted area and failure pressures of SHP wire screens in Section 2. We then discuss our custom-designed setup for testing a screen's breakthrough pressure in Section 3. In Section 4, we present available expressions for calculating the slip length over a SHP wire screen. Finally, our results and discussion are given in Section 5 followed by our conclusions in Section 6.

\section{Predicting Wetted AREA AND FAILURE PRESSURES}

In our simulations, sagging pressure, breakthrough pressure and slip length are calculated for simple squareweave wire screens (same number of wires per unit length in both directions). These screens are generally described by the geometric parameters: spacing between the wires $s_{w}$, diameter of the wires $d_{w}$, and the center to center distance between the wires $l_{w}$, as can be seen in Fig. 1. The Surface Evolver (SE), finite element code, is 
used in our study to determine the shape and position of the AWI under elevated hydrostatic pressures [36]. The screen is simulated by modeling a unit cell of the repeating geometry using symmetry boundary conditions on the planes slicing through the center line of the consecutive wires in the $x$ and $y$ directions (see Fig. 2a). The general form of energy equation for an AWI can be expressed as [37],

$E=p \iiint d V-\sum \sigma \cos \theta_{i} \iint d A_{i}$

where $p$ is the pressure difference across the interface, $\sigma$ is the surface tension, $\theta$ represents the YLCA. The surface energy associated with the wires depends on the water-solid surface area and it is described by the second term on the right hand side of Eq. 1. The area integral term in Eq. 1 should explicitly be defined for the SE. Figure $2 \mathrm{~b}$ shows the wetted area of a wire in a screen in the $x-y$ plane with the top of the wires placed at the $z=0$ plane. The AWI penetrates into the space between the wires in response to an increase in the water hydrostatic pressure. At the equilibrium position at an angle $\alpha$ from the top, the AWI is symmetrically draped over the top of the wire (see Fig. 2c). Note that the AWI touches the wire at point D making an angle $\alpha$ with the vertical axis (angle $B A D$ ). A wire oriented in the $x$-direction can mathematically be described as sine wave $\left[z-\frac{d_{w}}{2}\left(\sin \left(\frac{\pi}{l} x+\varphi\right)-1\right)\right]^{2}+y^{2}=\left(\frac{d_{w}}{2}\right)^{2}$ with $\varphi$ being the phase shift (in radian) from the origin (point A). For such a wire, the area element $d A_{i}$ can be written as

$d A_{i}=d_{w} \alpha F_{l} d x$

where $F_{l}=\sqrt{1+\left[f^{\prime}(x)\right]^{2}}$ with $f(x)=\frac{d_{w}}{2}\left[\sin \left(\frac{\pi}{l} x+\varphi\right)-1\right]$ is the z-coordinate of the wire centerline. Also, the angle $\alpha$ is a function of $f(x)$ according to [38]

$\alpha=\cos ^{-1}\left(1-2 f(x) / d_{w}\right)$ 
The above-mentioned equations (Eqs. 2-3) can be modified for the case where the wire is aligned in the $y-$ direction by replacing $x$ with $y$. Attention should be paid to how the volume integral term on the right hand side of Eq. 1 is defined for SE. To obtain the volume of the fluid above AWI correctly, one should subtract the volume of the portion of the wire $V_{S}$ (orange color) overlapping with the volume obtain by projecting the AWI onto the $x-y$ plane $(z=0)$ from AWI contact point (point $D$ ) as shown in Fig. 2c. Then, the volume of the fluid restricted by lines $C D$ and $A B$ above the wire $V_{F}$ (blue color) has to be added in the volume integral term. Finally, the volume integrand in Eq. 1 is modified by adding $V_{F}-V_{S}$ to become,

$$
d V=\left[-\frac{1}{8} d_{w}{ }^{2} \alpha+\frac{2 z+d_{w}}{8} d_{w} \sin \alpha\right] \sqrt{1+\left[f^{\prime}(x)\right]^{2}} d x
$$

Figures $3 \mathrm{a}-3 \mathrm{~d}$ show the simulated AWI over a wire screen under different hydrostatic pressures. The wires have an YLCA of $\theta=123^{\circ}$, a spacing of $s_{w}=458 \mu \mathrm{m}$, and a diameter of $d_{w}=254 \mu \mathrm{m}$. At low pressures, the meniscus merely touches the surface of the wires, but it penetrates deeper into the spacing between the wires as the pressure increases. In this work, we mainly focus on estimating the sagging pressure $P_{s a g}$ and breakthrough pressure $P_{b r k}$ for SHP screens with different dimensions. The sagging pressure $P_{s a g}$ is defined here as the pressure at which the AWI touches the flat substrate underneath the wire screen (Fig. 3c). The breakthrough pressure $P_{b r k}$ on the other hand, is defined here as the highest pressure that the AWI can tolerate before the AWI break up allowing water to flow through the screens (the maximum capillary pressure) as shown in Fig. 3d. As will be seen later in this paper, these pressures will be predicted for wire screens with different geometries and YLCAs. We also report the screens' wetted area at different hydrostatic pressures. We define the wetted area fraction $A_{w}$ of a screen as the ratio of the screen's wetted area from Eq. 1 to the unit cell area of the screen $l_{w}^{2}$. 
To ensure that the AWI predictions reported in this paper are not affected by the choice of mesh density, the wetted area fraction for a screen having a wire diameter of $d_{w}=140 \mu \mathrm{m}$, a wire spacing of $s_{w}=458 \mu \mathrm{m}$, and an YLCA of $\theta=123^{\circ}$ was calculated at different hydrostatic pressures using a uniformly distributed triangular mesh elements. The mesh density was then increased incrementally and its effects on the wetted area as well as sagging and breakthrough pressures were recorded. It was found that simulations performed with a mesh density of $L / d_{w}>500$ (where $L$ is the grid-points interval size) produce numerical results with less than about $5 \%$ mesh dependence (not shown for the sake of brevity).

\section{BREAKTHROUGH PRESSURE MEASUREMENT}

An experimental setup was built to determine the breakthrough pressure of the SHP wire screens for model validation. Four different wire screens were purchased from McMaster-Carr and cut into 2 inch by 2 inch samples. The screens were then sprayed with a commercially available hydrophobic coating (Ultra-Ever Dry by Ultratech Company). Prior to spraying the screens, the coating was applied on a glass slide and an average YLCA of 123 degrees was obtained (see Fig. 4). To coat the screens, an adhesive coating was first applied to each side of the screens using a sprayer bottle held 3 inches away and the screens were left to dry for 30 minutes. After the bottom coat had sufficiently dried, a top coat was sprayed on using another sprayer. The coating was sprayed in a fine mist and in a consistent way to maximize the uniformity of the coating to the extent possible. Attention was also paid to not apply excessive amount of coating onto the screens so that their geometry remain unaffected. Nevertheless, differences in the amount of coating applied to the various screens could account for some minor aberrations in the results. The screens were left to dry for 24 hours before being used in the experiment. Several screens of each size to be tested were coated to obtain statistically meaningful measurements. Occasionally, a film of hydrophobic coating would form a film across one or numerous pores in the screen. These screens were excluded from the experiment to create consistency and accuracy in the experimental data. 
To measure a screen's breakthrough pressure, a test setup was designed and built (see Fig. 5a). The setup consists of a vessel made of an acrylic cylinder that is fed with water at a defined pressure. The pressure of water is adjusted by changing the height of the water in a tube. The top of the chamber is comprised of the holder where the screen can be mounted. The screen was placed on top of the vessel and then a hydrophobic o-ring centered on the hole in the middle of the plate. A clear circular plate was then placed on top of the o-ring and the screen before fastening the top plate to the rest of the vessel. The height gauge was adjusted until the water level reached the surface of the screen and the manometer level became stable. The height gauge was then zeroed and the microscope was positioned over the pressure vessel. A possible variation in the results could have resulted from inconsistencies in this zeroing because it was a judgment based on visual observation and could vary slightly from trial to trial. The level of the height gauge was slowly elevated until it was observed through the microscope that the water had penetrated the hydrophobic coating of the screen. The elevated hydrostatic pressure could then be calculated as $P_{b r k}=\rho g h$ where $\rho$ is the density of water, $g$ is the gravitational acceleration, and $h$ is the height of the water column in the tube. The maximum water (dyed with food coloring to allow a more definable interface) level that a SHP screen can withstand without water breaking through was measured optically [21]. The AWI breaking through the screen can be seen in Figs. 5b and 5c. In order to eliminate the potential edge effects, results were only considered from trials in which the water penetrated the screen from a pore that was not directly touching the pressure vessel. The screen was removed and the vessel was dried so that the next trial can be conducted. The screen, o-ring and both flanges had to be completely dried in order for an accurate trial to be conducted.

\section{SLIP LENGTH PREDICTION}

\subsection{FLOW FiELD CALCULATION}


As mentioned earlier, the AWI formed over the pores of a SHP surface can provide drag reduction benefits for a submerged surface. A body of water flowing over a SHP wire screen experiences frictional (no-slip) contact with the solid wires and slippery (no shear) contact along the AWI (see Fig 6a). Overall, one can expect a reduction in the skin-friction drag of a solid surface due to a reduction in the surface wetted area upon using a SHP screen. The decrease in the skin friction drag is often portrayed by the effective slip length, which is the average distance underneath the water-solid interface at which the velocity extrapolates to zero (refer Fig. 6b). As discussed before in Sec. 2, the shape and position of the AWI over a SHP wire screen can be obtained from SE simulations at different pressures. This information can be used to predict the slip length over such surfaces by solving the Navier-Stokes equation in a Couette geometry such as the one shown in Fig. 6 (note the periodic and symmetric boundary conditions in the streamwise and lateral directions, respectively). A laminar flow is induced by moving the upper plate with a velocity of $0.1 \mathrm{~m} / \mathrm{s}$ in the $x$-direction. Unless otherwise stated, the distance between the SHP surface and the upper plate is considered to be $H=5 d_{w}$ in all the simulations reported here. The flow field is obtained using the finite volume FLUENT software. The computational domain was meshed using tetrahedral mesh refined near the AWI to reduce the total number of volume mesh (i.e., CPU time) required to obtain a reliable flow field between the plates. In addition, regional mesh adaption was considered near the AWI or the upper plate whenever it showed to further improve the slip length predictions. A mesh-independence study was also conducted (with $d_{w}=140 \mu \mathrm{m}, s_{w}=458 \mu \mathrm{m}$, and YLCA $\theta=123^{\circ}$ at a hydrostatic pressure of $100 \mathrm{~Pa}$ ) to examine how our predicted effective slip length values vary with the total number of volume mesh $N$ used in our flow field simulations (not shown for the sake of brevity). It was found that simulations conducted with more than 1.5 million tetrahedral mesh (with the near-AWI refinement) had negligible dependence on the total number of volume mesh. Additional regional mesh adaptation was considered whenever needed to further improve the accuracy of our slip length calculations.

\subsection{SLIP LENGTH CALCULATION AND VALIDATION}


The most practical way of defining an effective slip length is what one may observe in a rheometer experiment with the SHP wire as the bottom plate. In this configuration, the drag benefits of a SHP wire can be examined by the reduced torque (shear stress) needed to spin the upper plate [9],

$$
\bar{b}_{u p}=\left(\frac{\tau_{f l a t}}{\tau_{\text {slip }}}-1\right) H
$$

where $\tau_{\text {flat }}$ is the conventional Couette flow shear stress at the upper plate (in the absence of a SHP bottom plate), and $\tau_{s l i p}$ is the shear stress at the upper plate with the SHP wire screen as the lower plate. An approximate analytical effective slip length expression is also provided in [8] for flow over SHP screens, as

$$
\bar{b}_{a p p} \approx \frac{l_{w}}{3 \pi} \ln \left(\frac{2\left(1+\sqrt{1-A_{w}}\right)}{\pi A_{w}}\right)
$$

According to this equation, effective slip length is positive for $A_{w}<0.868$, and negative when $0.868<A_{w} \leq 1$. To the knowledge of the authors, the only analytical work that can be used to validate our numerical slip length calculations over SHP wire screens is the work of [8] who proposed an effective slip length expression as,

$$
\bar{b}_{f l a t}=l_{w}\left(a_{1} \ln \varepsilon+a_{2}\right)
$$

where $\varepsilon=d_{w} / l_{w}, a_{1}=-0.107$, and $a_{2}=-0.069$, with the solid area fraction defined as $A_{w}=\varepsilon(2-\varepsilon)$. The effective slip length expression of [8] treats the wire screen as a 2-D planar surface (i.e., no curvatures is considered for the AWI or the wires). Therefore, our simulation domain was simplified to numerically duplicate the geometry of screen as considered in [8]. The simulation domain and the resulting slip length values from Eq. 5 are given in Fig. 7 along with those of Eqs. 6 and 7. For these calculations wire screens with a unit cell length of $l_{w}=636 \mu \mathrm{m}$ but varying solid area fractions of $0.19<A_{w}<0.75$ were considered. Good agreement can be seen between the three approaches taken to predict an effective slip length for flow over a SHP wire screen treated as a 
2-D geometry comprised of flat wires accommodating a flat AWI (i.e., a flat plate perforated with square holes having sharp edges).

\section{RESULTS AND DISCUSSION}

In this section, we discuss how diameter and spacing of the wires in a screen can impact a screen's sagging and breakthrough pressures as well as its wetted area and slip length. We will compare the results of numerical simulations with those obtained from our experiment whenever possible.

Figure 8 shows the effects of wire spacing on sagging and breakthrough pressures for screens with different wire diameters of $d_{w}=254$ and $508 \mu \mathrm{m}$. It can be seen that both pressures decrease when increasing the spacing between the wires. The decreasing trend can be better understood by considering the fact that the AWI contact line (providing capillary forces) scales almost linearly with the wire spacing whereas AWI area (exposed to hydrostatic pressure) scales with the wire spacing squared. Therefore, increasing wire spacing weakens the resistance of a screen to an intruding AWI. More interesting in this figure is the difference between the breakthrough and sagging pressures. While in most cases sagging pressure is smaller than the breakthrough pressure, it is quite possible for this trend to reverse especially at small wire spacing values (i.e., AWI gives in before it comes into contact with the underlying substrate) as shown with blue shade in Fig. 8a and Fig. 8b for $s_{w}<254 \mu \mathrm{m}$ and $s_{w}<762 \mu \mathrm{m}$, respectively. In such cases, the breakthrough pressure is taken as the failure pressure. Figure 8 also shows the effects of wire spacing on the screens' wetted area fraction at sagging and breakthrough pressures. It can be seen that wetted area fractions decrease with increasing wire spacing. This is 
because the unit cell area of a screen increases faster with increasing wire spacing than does the wires' wetted area. The breakthrough pressures obtained from our experiments are shown in Fig. $8 \mathrm{a}\left(s_{w}=660 \mu \mathrm{m}\right.$ and $s_{w}=1448 \mu \mathrm{m}$ ) and Fig. $8 \mathrm{~b}$ (and $s_{w}=762 \mu \mathrm{m}$ and $s_{w}=1296 \mu \mathrm{m}$ ) with red symbols. The experimental data seem to follow the numerical predictions slightly better for screens with larger wire spacing. This appears to be caused by the inaccuracies involved in tracking the AWI experimentally (i.e., optically) when the wire spacing is smaller.

Comparing screens with identical wire spacing but different wire diameters in Fig. 8, it can be seen that both the sagging and breakthrough pressures are generally higher for screens with bigger wire diameters especially when the spacing is large. For instance, for a wire spacing of $s_{w}=1446 \mu \mathrm{m}$, the sagging and breakthrough pressures are found respectively to be $P_{s a g}=102 \mathrm{~Pa}$ and $P_{b r k}=138 \mathrm{~Pa}$, for the screen having a wire diameter of $d_{w}=254 \mu \mathrm{m}$ (Fig. 8a), and $P_{s a g}=135 \mathrm{~Pa}$ and $P_{b r k}=160 \mathrm{~Pa}$ for the screen with the wire diameter of $d_{w}=508 \mu \mathrm{m}$ (Fig. 8b). For a smaller spacing of $s_{w}=746 \mu \mathrm{m}$ however, these pressures are predicted to be $P_{\text {sag }}=275 \mathrm{~Pa}$ and $P_{b r k}=300 \mathrm{~Pa}$ for the screen with the wire diameter of $d_{w}=254 \mu \mathrm{m}$, and $P_{s a g}=P_{b r k}=300 \mathrm{~Pa}$ were for screen with the wire diameter of $d_{w}=508 \mu \mathrm{m}$.

Figure 9 shows the effects of pressure on wetted area fraction and effective slip length of wire screens with different geometries. Two sets of data are generated for this study; one in which the spacing is varied, while the wire diameter is held constant at $d_{w}=254 \mu \mathrm{m}$ (Figs. 9a and 9b), and the other where spacing is fixed at $s_{w}=178 \mu \mathrm{m}$ but the wire diameter is varied (Figs. 9c and 9d). As expected, wetted area fraction increases with pressure for both cases (see Fig. 9a and 9c). More interestingly, at a constant pressure (say, $P=150 \mathrm{~Pa}$ ), wetted area fraction is found to be larger for screens with larger wire diameters or smaller wire spacing. This information is used in Eq. 6 to predict the effective slip length of the surface. Effective slip length can also be predicted (more accurately) using Eq. 5 after solving the Navier-Stokes equations in a Couette geometry. Figures 9b and 9d compare the slip length predictions of these two equations with one another. It can be seen that Eq. 5 predicts a 
decreasing slip length which reaches a plateau beyond some pressure (around $100 \mathrm{~Pa}$ in Fig. $9 \mathrm{~b}$ and $250 \mathrm{~Pa}$ in Fig. 9d). To better explain this, velocity vectors in a plane slicing through the wire screen (the case with a wire diameter of $d_{w}=140 \mu \mathrm{m}$ and a wire spacing of $s_{w}=178 \mu \mathrm{m}$ ) are shown in Fig. 10a and their average values at a distance $\delta$ above the surface are calculated for operating pressures of 0 and $500 \mathrm{~Pa}$ (see the dashed line in the inset figure). It can be seen that, average velocity above the surface is almost independent of operating pressure, even when the AWI penetrates deeper into the screen when the pressure is higher. This means despite the increase in the wetted area of the screens under pressure, no measurable additional friction is generated against the flow. This observation is also consistent with the results reported in [39].

The results given in Figs. 9b and 9d indicate that slip length decreases continuously with pressure when predicted using the approximate formula given in Eq. 6 (even reaching negative slip length values). However, this equation seems to provide reasonable predictions at relatively low pressures, and more importantly, it does not require solving the Navier-Stokes equation. Figure $9 \mathrm{~b}$ shows that, at a given operating pressure, effective slip length is higher for wire screens with larger wire spacing. This is mostly due to the fact that wetted area fraction is smaller for such screens (see Fig. 9a). On the contrary, Fig. 9d shows that, at a given pressure, effective slip length over wire screens with identical wire spacing is higher for screens with larger wire diameters. This is in contradiction with the wetted area fraction results shown in Fig. 9b. However, it should be noted that the results shown in Fig. 9d were obtained for a fixed gap of $H=1000 \mu \mathrm{m}$ between the upper and lower plates. Therefore, the effective gap (distance between the top surface of the wires and the moving upper wall) decreases with increasing the wire diameter, and this makes the screen with a larger wire diameter produce more slip length despite having a larger wetted area fraction. To further investigate this, effective slip length over a screen with a wire diameter of $d_{w}=254 \mu \mathrm{m}$ and a wire spacing of $s_{w}=305 \mu \mathrm{mis}$ calculated for two different gap distances of $H=1270 \mu \mathrm{m}$ and $H=635 \mu \mathrm{m}$ in Fig. 10b. It can be seen that slip length increases by about $20 \%$ when the gap is decreased by a factor of 2. This is in qualitative agreement with the observation reported in [40]. 


\section{CONCLUSIONS}

Woven screens enhanced with functional surface treatments/coatings have recently be considered as a costeffective alternative for producing a SHP porous surface in many applications including but not limited to drag reduction [8-11], oil-water separation [12-16], self-cleaning and anti-icing [17-19], and device manufacturing [24-28] among many others. In this work, we reported on the failure pressure of such screens and discuss how the interplay between the wire diameter and wire-to-wire spacing can affect the performance of a SHP screen. An approach for obtaining an accurate estimate of a screen's wetted area in 3-D is presented and is used to calculate its drag reduction benefit (slip length) when the screen is used in a Couette flow configuration. Our simulations indicate that operating pressure in the Couette configuration does not significantly affect the drag reducing effects of the screens. Different formulations proposed in the literature for predicting the slip length of a SHP surface are compared with one another and their pros and cons are discussed in detail.

\section{ACKNOWLEDGMENTS}

The authors acknowledge partial support from the National Science Foundation CMMI (1029924) and CBET (1402655) programs. 


\section{REFERENCES}

[1] D. Quere, Wetting and roughness, Annu. Rev. Mater. Res. 38 (2008) 71-99.

[2] N.J. Shirtcliffe, G. McHale, S. Atherton, M.I. Newton, An introduction to superhydrophobicity, Adv. Colloid Interf. Sci. 161 (2010) 124-138.

[3] J.P. Rothstein, Slip on superhydrophobic surfaces, Annu. Rev. Fluid Mech. 42 (2010) 89-109.

[4] J. Ou, J. P. Rothstein, Direct velocity measurements of the flow past a drag-reducing utrahydrophobic surfaces, Phys. Fluids 17 (2005) 103606.

[5] C. Lee, C.-H. Choi, C.-J. Kim, Structured surfaces for giant liquid slip, Phys. Rev. Lett. 101 (2008) 064501.

[6] M. A. Samaha, H.V. Tafreshi, M. Gad-el-Hak, Effects of hydrostatic pressures on drag-reduction performance of submerged aerogel particle coatings, Colloids Surf. A 399 (2012) 62-70.

[7] A. Hemeda, H.V. Tafreshi, Instantaneous Slip-Length in Superhydrophobic Microchannels: Grooves with Dissimilar Walls or Arbitrary Wall Curvatures, Phys. Fluids 27 (2015) 102101.

[8] A. M. J. Davis, E. Lauga, The friction of a mesh-like super-hydrophobic surface, Phys. Fluids 21 (2009) 113101.

[9] S. Srinivasan, W. Choi, K.C. Park, S.S. Chhatre, R.E. Cohen, G.H. McKinley, Drag reduction for viscous laminar flow on spray-coated non-wetting surfaces, Soft Matter 9 (2013) 5691-5702.

[10] J.C. Brennan, N.R. Geraldi, R.H. Morris, D.J. Fairhurst, G. McHale, M.I. Newton, Flexible conformable hydrophobized surfaces for turbulent flow drag reduction, Sci. Rep. 5 (2015) 10267 .

[11] S. Srinivasan, J.A. Kleingartner, J.B. Gilbert, R.E. Cohen, A.J.B. Milne, G.H. McKinley, Sustainable Drag Reduction in Turbulent Taylor-Couette Flows by Depositing Sprayable Superhydrophobic Surfaces, Phys. Rev. Lett. 114 (2015) 014501.

[12] C. H. Lee, N. Johnson, J. Drelich, Y. K. Yap, The performance of superhydrophobic and superoleophilic carbon nanotube meshes in water-oil filtration, 49 (2011) 669-676.

[13] J. Song, S. Huang, Y. Lu, X. Bu, J.E. Mates, A. Ghosh, R. Ganguly, C.J. Carmalt, I.P. Parkin, W. Xu, C.M. Megaridis, Self-driven one-step oil removal from oil spill on water via selective-wettability steel mesh, ACS Appl. Mater. Interfaces 6 (2014) 19858-19865.

[14] J. Li, L. Yan, H. Li, J. Li, F. Zha, Z. Lei, A facile one-step spray-coating process for the fabrication of a superhydrophobic attapulgite coated mesh for use in oil/water separation, RSC Adv. 5 (2015) 53802.

[15] S.U. Patel, G.G. Chase, Separation of water droplets from water-in-diesel dispersion using superhydrophobic polypropylene fibrous membranes, Separ. Purif. Technol. 126 (2014) 62-68.

[16] S.U. Patel, S.U. Patel, G.G. Chase, Electrospun superhydrophobic poly(vinylidene fluoride-cohexafluoropropylene) fibrous membranes for the separation of dispersed water from ultralow sulfur diesel, Energy Fuels 27 (2013) 2458-2464. 
[17] C.W. Extrand, Repellency of the lotus leaf: resistance to water intrusion under hydrostatic pressure, Langmuir 27 (2011) 6920-6925.

[18] A. Ganne, V.O. Lebed, A.I. Gavrilov, Combined wet chemical etching and anodic oxidation for obtaining the superhydrophobic meshes with anti-icing performance, Colloids Surf. A 499 (2016) 150-155.

[19] E.J.Y Ling, V Uong, J-S. Renault-Crispo, A-M. Kietzig, P Servio, Reducing Ice Adhesion on Nonsmooth Metallic Surfaces: Wettability and Topography Effects, ACS Appl. Mater. Interfaces 8 (2016) 8789-8800.

[20] S.M. Lee, D.J. Oh, I. D. Jung, P. G. Jung, K. H. Chung, W. I. Jang, J. S. Ko, Evaluation of the waterproof ability of a hydrophobic nickel micromesh with array-type microholes, J. Micromech. Microeng. 19 (2009) 125024.

[21] Y.M. Shin, S.K. Lee, J.Y. Lee, J.H. Kim, J.H. Park, C.H. Ji, Microfabricated environmental barrier using $\mathrm{ZnO}$ nanowire on metal mesh, J. Micromech. Microeng. 23 (2013) 127001.

[22] K.-C Park, S.S. Chhatre, S. Srinivasan, R.E. Cohen, G.H. McKinley, Optimal Design of Permeable Fiber Network Structures for Fog Harvesting, Langmuir 29 (2013) 13269-13277.

[23] N.R. Geraldi, G. McHale, B.B. Xu, G.G. Wells, L.E. Dodd, D. Wood, M.I. Newton, Leidenfrost transition temperature for stainless steel meshes, Mater. Lett. 176 (2016) 205-208.

[24] Z.X. Jiang, L. Geng, Y.D. Huang, Design and fabrication of hydrophobic copper mesh with striking loading capacity and pressure resistance, J. Phys. Chem. C 114 (2010) 9370-9378.

[25] Z.X. Jiang, L. Geng, Y.D. Huang, Fabrication of superhydrophobic 3-D braided carbon fiber fabric boat, Mater. Lett. 64 (2010) 2441-2443.

[26] Q. Pan, M. Wang, Miniature boats with striking loading capacity fabricated from superhydrophobic copper meshes, ACS Appl. Mater. Interfaces 1 (2009) 420-423.

[27] Z.X. Jiang, L. Geng, Y.D. Huang, S.A. Guan, W. Dong, Z.Y. Ma, The model of rough wetting for hydrophobic steel meshes that mimic Asparagus setaceus leaf, J. Colloid Interface Sci. 354 (2011) 866-872.

[28] S.S. Chhatre, W. Choi, A. Tuteja, K-C Park, J.M. Mabry, G.H. McKinley, R.E. Cohen, Scale Dependence of Omniphobic Mesh Surfaces, Langmuir 26 (2010) 4027-4035.

[29] B. Emami, H.V. Tafreshi, M. Gad-el-Hak, G.C. Tepper, Effect of Fiber Orientation on Shape and Stability of Air-Water Interface on Submerged Superhydrophobic Electrospun Thin Coatings, J. Appl. Phys. 111 (2012) 064325 .

[30] T.M. Bucher, B. Emami, H.V. Tafreshi, M. Gad-el-Hak, G.C. Tepper, Resistance of NanoFibrous Superhydrophobic Coatings to Hydrostatic Pressure: the Role of Microstructure, Phys. Fluids 24 (2012) 022109.

[31] C.W. Extrand, Criteria for Ultralyophobic Surfaces, Langmuir 20 (2004) 5013-5018.

[32] B. Emami, T.M. Bucher, H.V. Tafreshi, D. Pestov, M. Gad-el-Hak, G.C. Tepper, Simulation of meniscus stability in superhydrophobic granular surfaces under hydrostatic pressure, Colloids Surf., A 385 (2011) 95-103

[33] A. Rawal, Design Parameters for a Robust Superhydrophobic Electrospun Nonwoven Mat, Langmuir 28 (2012), 3285-3289. 
[34] M.M. Amrei, H.V. Tafreshi, Effects of hydrostatic pressure on wetted area of submerged superhydrophobic granular coatings. Part 1: mono-dispersed coatings, Colloids Surf., A 465 (2015) 87.

[35] A. Tuteja, W. Choi, J.M. Mabry, G.H. McKinley, R.E. Cohen, Robust omniphobic surfaces, Proc. Natl. Acad. Sci. USA, 105 (2008) 18200.

[36] K.A. Brakke, The surface evolver and the stability of liquid surfaces, Philos. Trans. R.Soc. Lond. A 354 (1996) 2143-2157.

[37] P.G. de Gennes, F. Brochard-Wyart, D. Quéré, Capillarity and Wetting Phenomena: Drops, Bubbles, Pearls, Waves, Springer-Verlag, New York, 2004.

[38] T.M. Bucher, M.M. Amrei, H.V. Tafreshi, Wetting resistance of heterogeneous superhydrophobic coatings with orthogonally layered fibers, Surf. Coat. Technol. 277 (2015) 117-127.

[39] A. S. Haase, E. Karatay, P. A. Tsai, R.G.H. Lammertink, Momentum and mass transport over a bubble mattress: the influence of interface geometry, Soft Matter 9 (2013) 8949-8957.

[40] X. Yang, Z. C. Zheng, Effects of channel scale on slip length of flow in micro/nanochannels, J. Fluids Eng. 132 (2010) 061201-061206. 


\section{NOMENCLATURE}

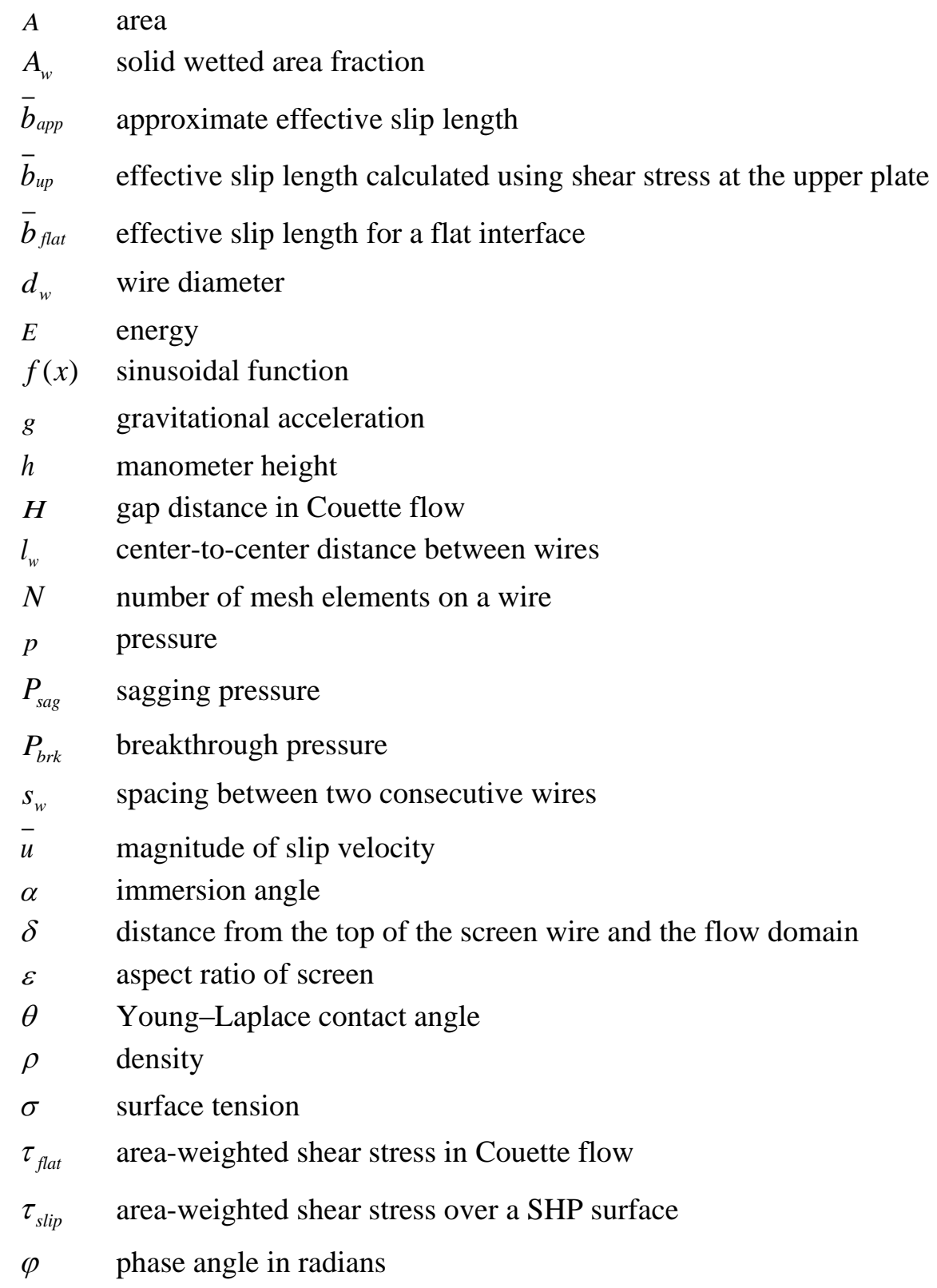

\section{ABBREVIATIONS}

AWI air-water interface

CHP critical hydrostatic pressure

PBC periodic boundary conditions

SE surface evolver

YLCA Young-Laplace contact angle 


\section{FIGURE CAPTIONS}

Figure 1: An example SEM image of the superhydrophobic wire screens considered in the study.

Figure 2: Virtual unit-cell of a wire screen used in SE calculations (a). The area (b) and volume (c) elements used in deriving the energy equation for the system.

Figure 3: Sample simulation results showing the AWI under different hydrostatic pressures in (a) and (b). Failure due to AWI sagging is shown in (c). Failure due to AWI breakup at the breakthrough pressure is shown in (d). Here

$d_{w}=254 \mu \mathrm{m}$ and $s_{w}=458 \mu \mathrm{m}$.

Figure 4: An SEM image of the superhydrophobic spray-on coating used on a microscope slide. The inset shows droplet contact angle measured via a goniometer.

Figure 5: Our experimental setup designed to measure the breakthrough pressure of SHP woven screens (a). Optical images of AWI trying to enter a SHP screen at low (b) and high (c) magnifications.

Figure 6: The computational domain considered for solving the Navier-Stokes equations using an accurate estimate of the 3-D shape of the AWI over the screens (a). Schematic diagram describing the slip length concept (b).

Figure 7: Dimensionless effective slip length values calculated for screens with different area fractions. An oversimplified representation of the screens geometry in considered here to compare the results of our numerical calculations with those of [8] for validation purposes.

Figure 8: Effects of wire-to-wire spacing $s_{w}$ on failure pressure (breakthrough and sagging pressures) and wetted area fraction of the screens having constant wire diameters of $d_{w}=254 \mu \mathrm{m}$ (a) and $d_{w}=508 \mu \mathrm{m}$ (b). Experimental breakthrough pressure values (red symbols) are added to the figures for comparison.

Figure 9: Effects of hydrostatic pressure wetted area fraction ( $a$ and $c$ ) and slip length ( $b$ and d) for screens with different geometries. Slip length predictions of Eq. $5\left(\bar{b}_{u p}\right)$ and Eq. $6\left(\bar{b}_{a p p}\right)$ are compared with one another.

Figure 10: Average velocity at a distance $\delta$ above the solid surface in a plane slicing through the screen is shown for two different hydrostatic pressures of 0 and $500 \mathrm{~Pa}$ in (a) along with example velocity profiles. Effects of the gap distance between the upper and lower plate in Couette geometry $H$ on slip length is shown in (b) for two different gap values as a function of pressure. 


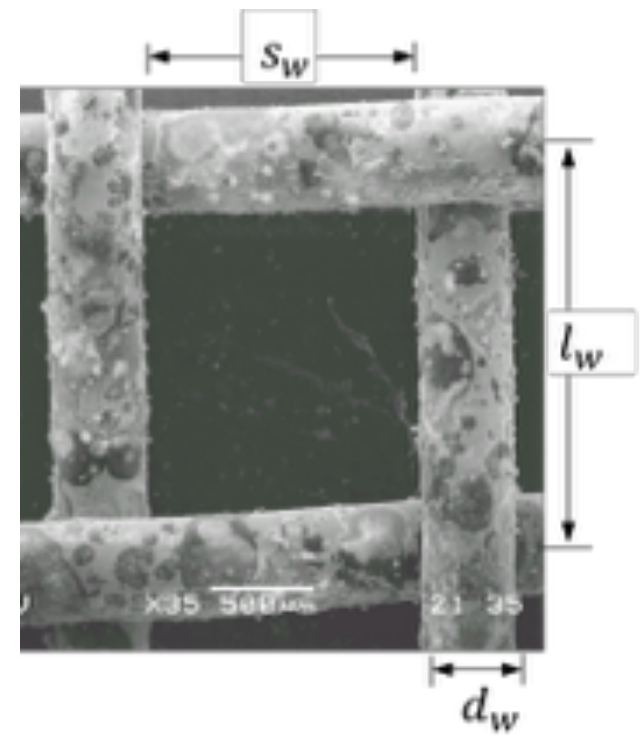

Figure 1: 
(a)

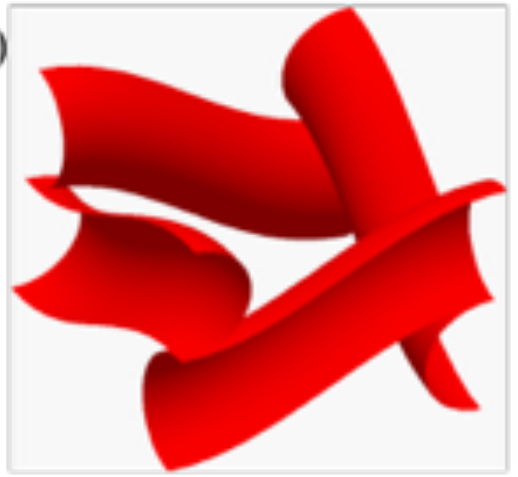

(b)
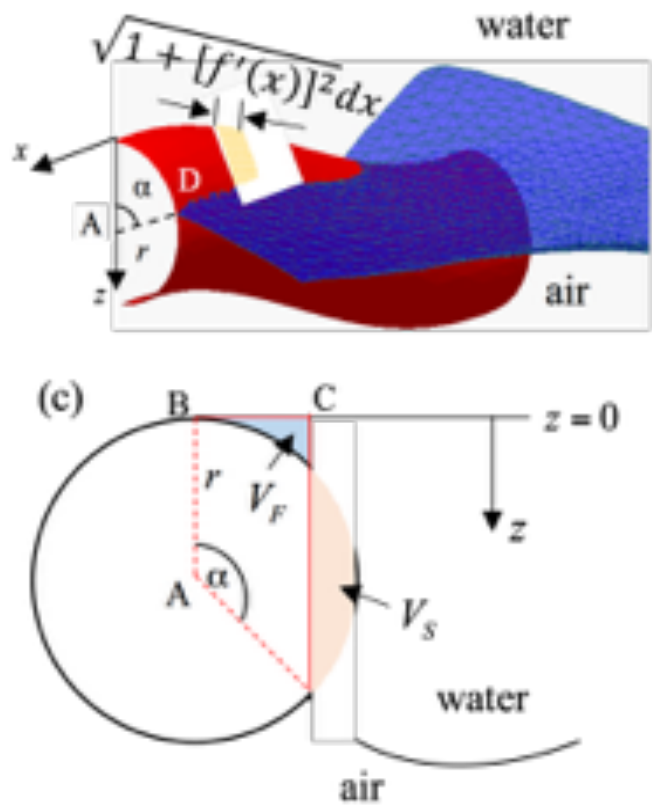

Figure 2: 


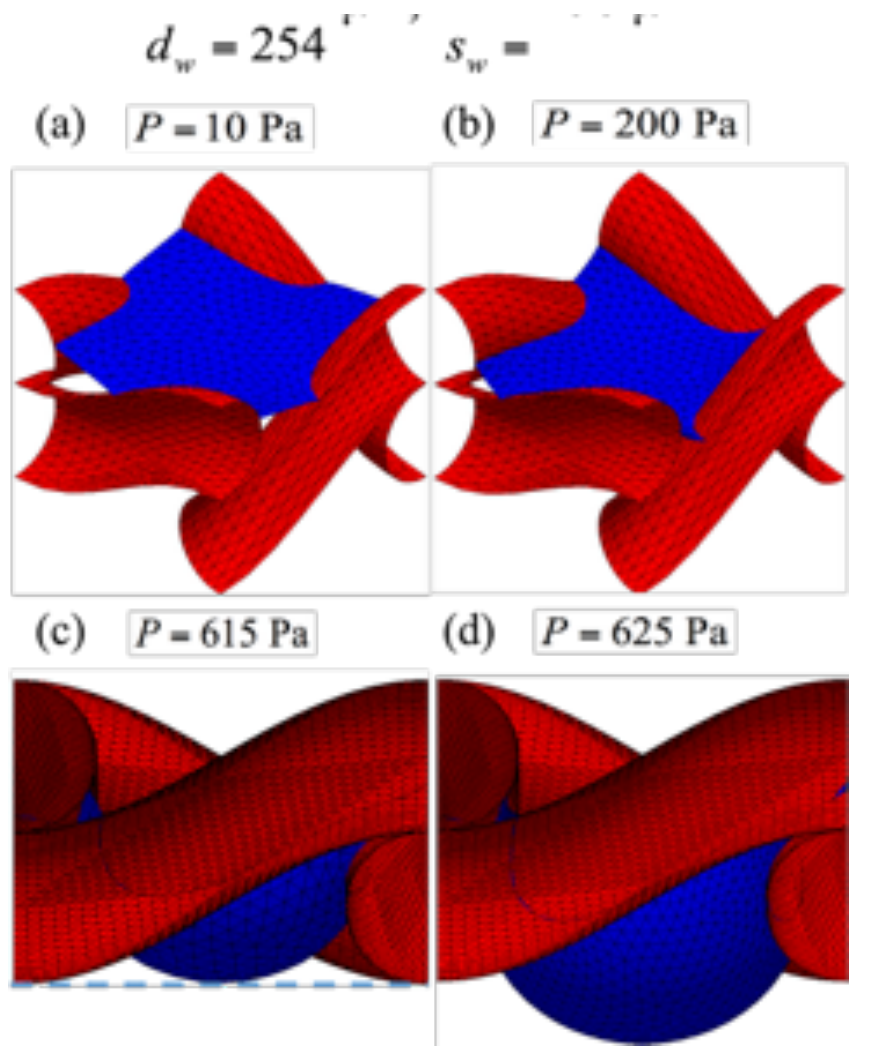

Figure 3: 


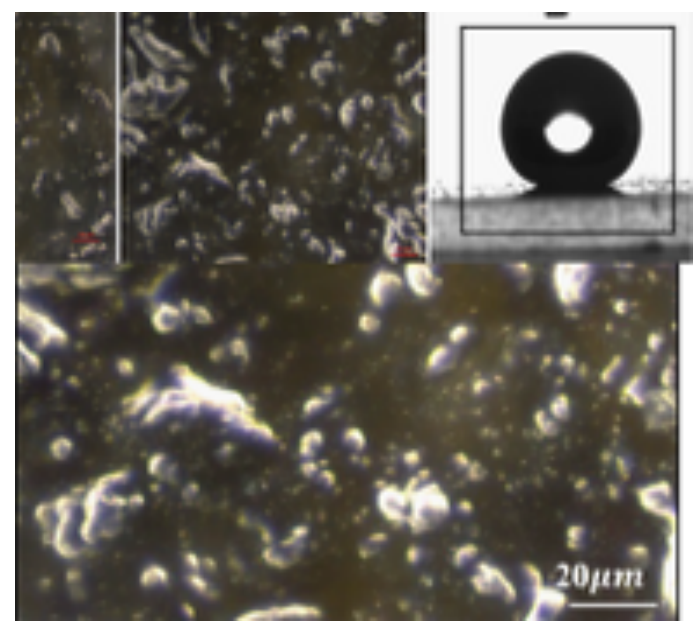

Figure 4: 


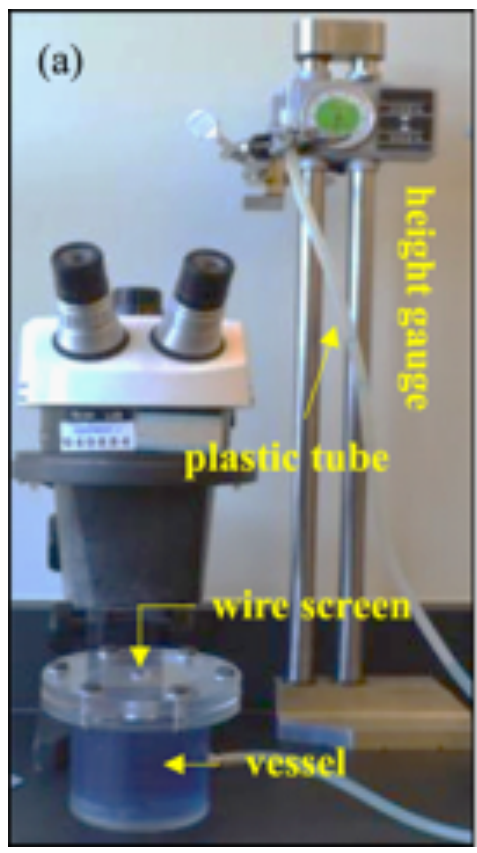

(b)

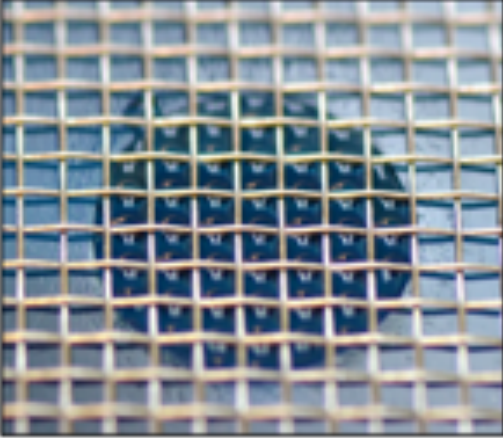

(c)

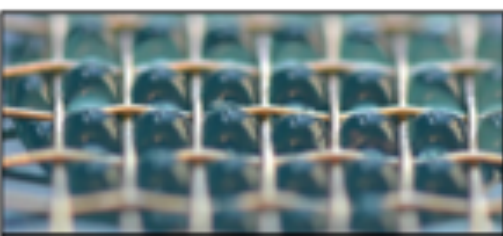

Figure 5: 




Figure 6: 


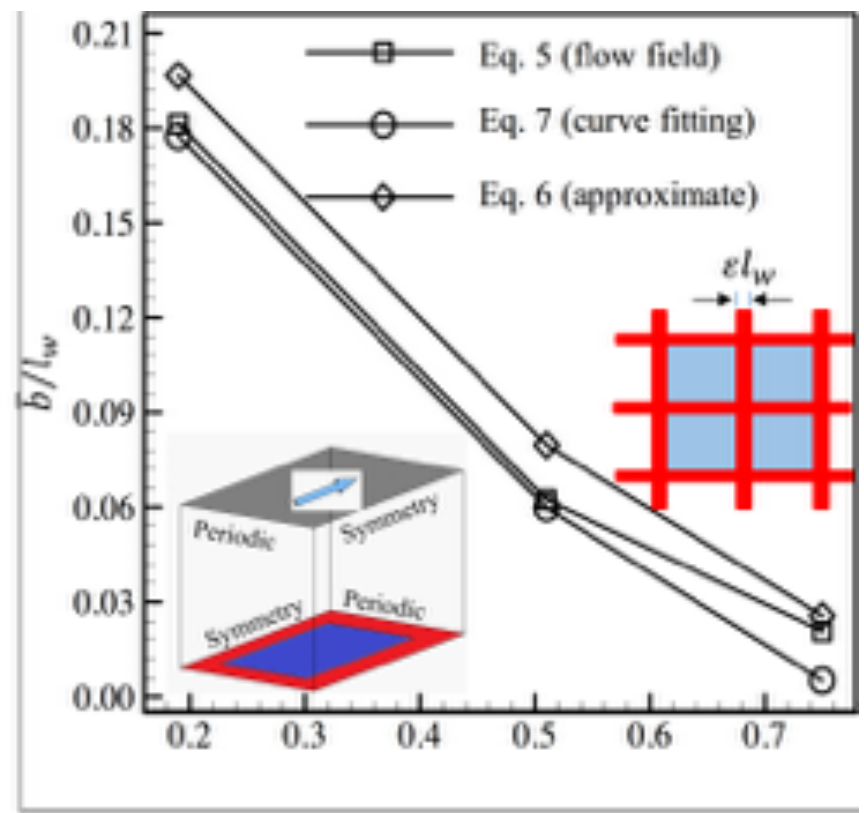

Figure 7: 


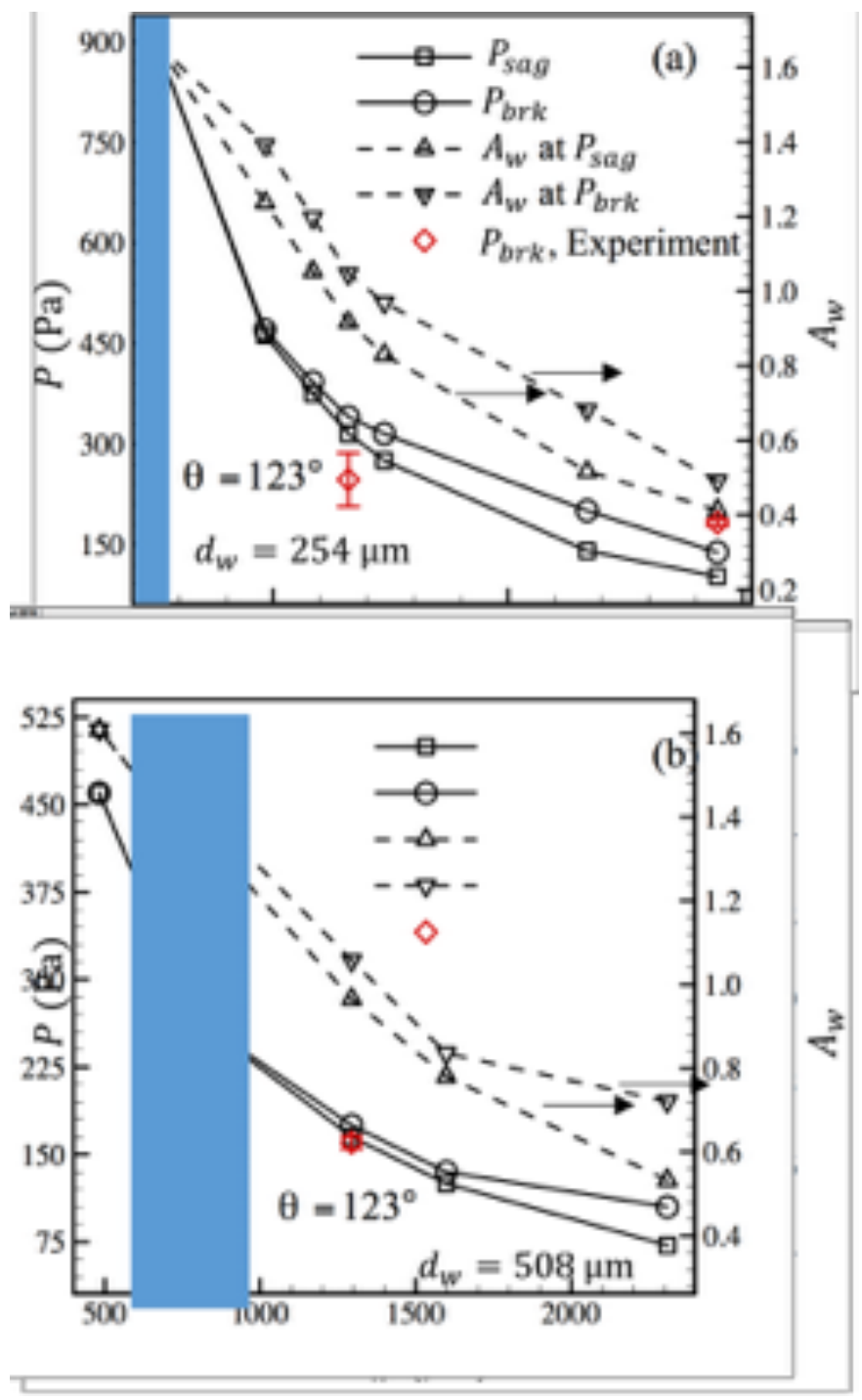

Figure 8: 


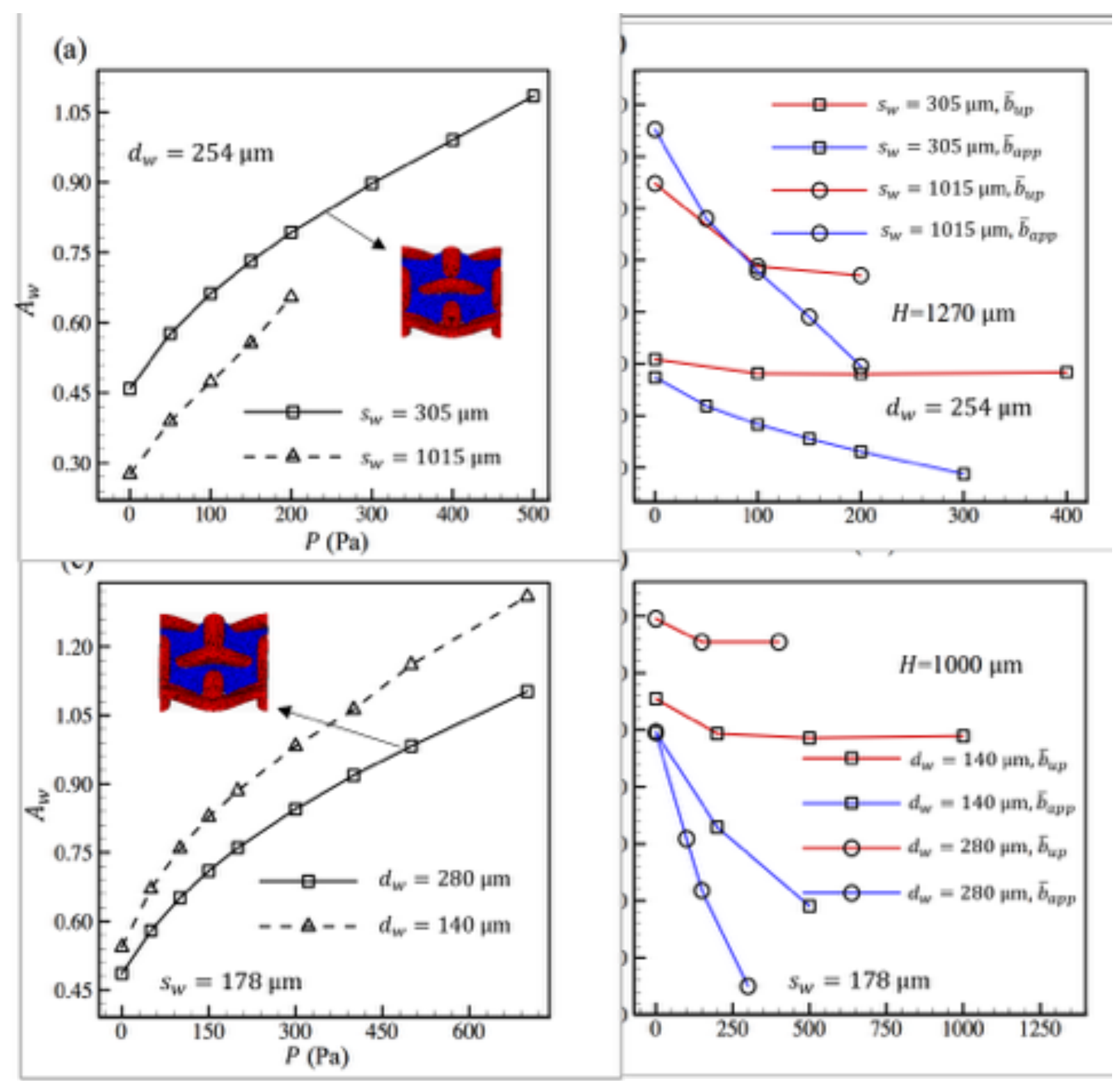

Figure 9: 


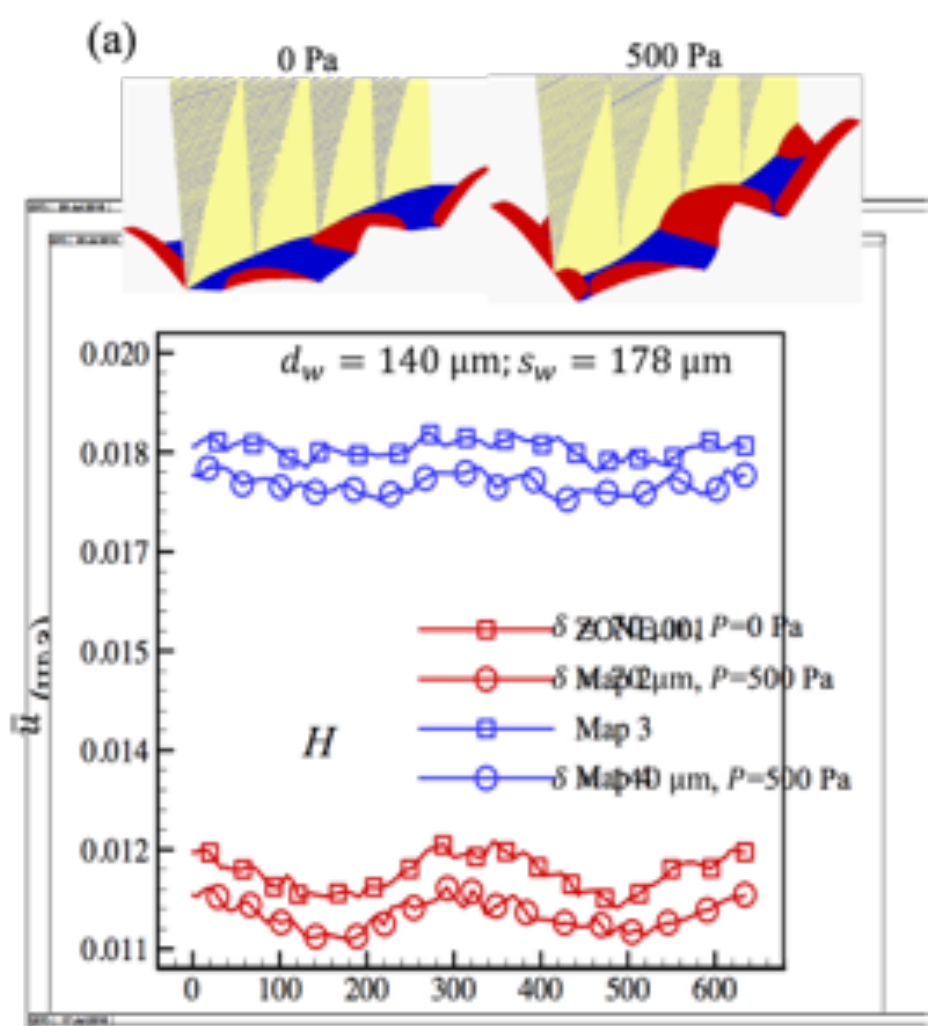

(b)

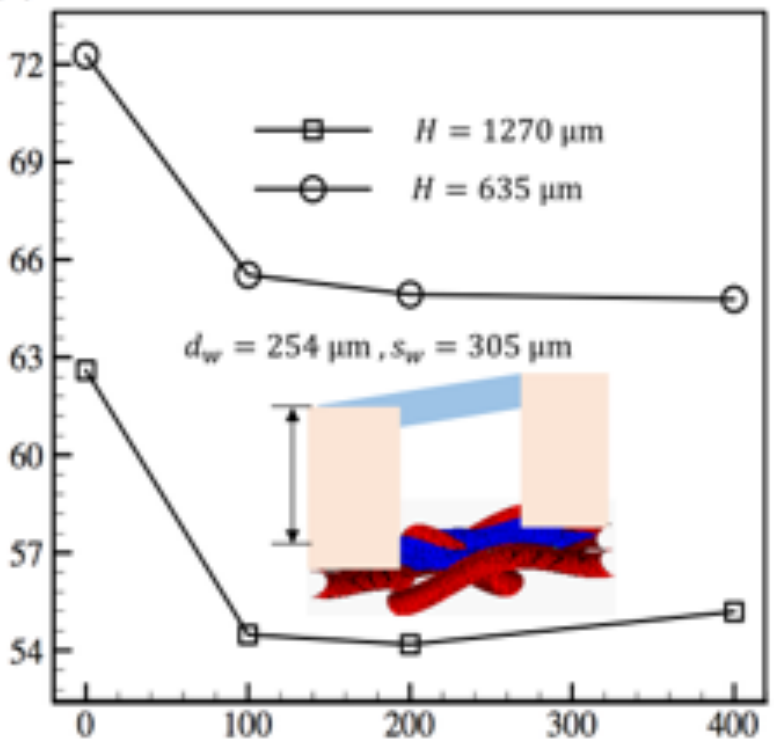

Figure 10: 
Graphical Abstract

Failure Pressure and Drag Reduction Benefits of Superhydrophobic Woven Screens
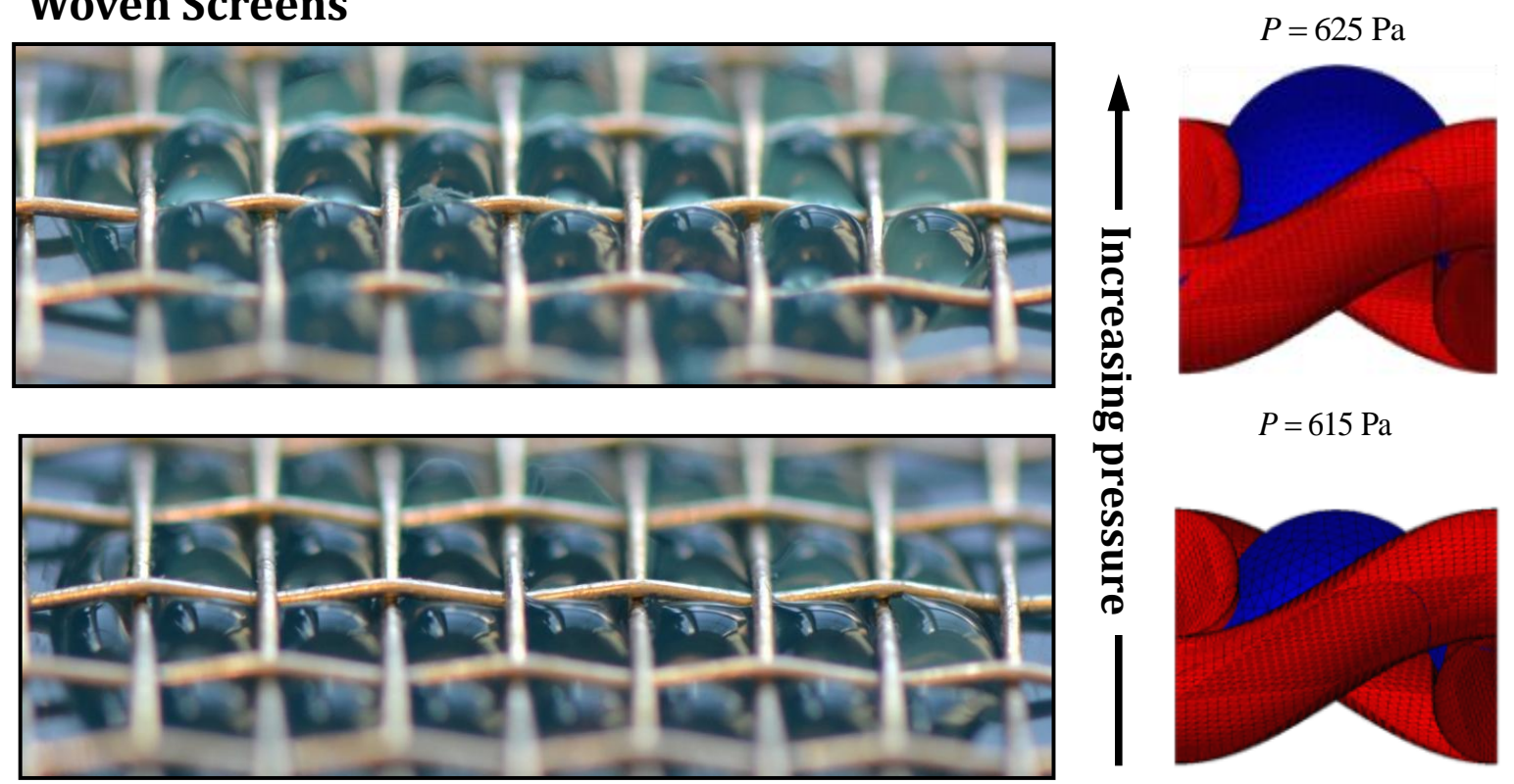